\title{
Study on the Engineering Simulation of the Electric Hot Air De-icing
}

\section{Device of the Substation Equipment}

\author{
Jian $\mathrm{Li}^{1, \mathrm{a}}$ \\ ${ }^{1}$ Department of Science and Technology, State Grid Electric Power Research Institute, Nanjing, \\ Jiangsu Prov, China \\ a15171495741@163.com
}

Keywords: Frozen disaster, substation equipment, hot air de-icing, engineering calculation

Abstract: In this paper, heat characteristics, energy conversion efficiency and energy transmission distance of electric hot air de-icing device are researched. Thermal energy conversion formula of jet bushing of hot air device is obtained. Finite element analysis software is used to establish the computing model of hot air de-icing device such as bushing shell, switches and disconnecting link. Take the bushing shell for $500 \mathrm{kv}$ transformer for example, de-icing performance is simulated, and de-icing time, energy consumption and energy utilization efficiency are analyzed in this paper. Feasibility and effectiveness of the electric hot air de-icing device is validated too.

\section{Introduction}

It is well known that safe operation of the power grid is very important. In recent years, winter snow freeze disaster has resulted in failure of power grid operation. Especially in early 2008, the most areas of southern China appeared a rare heavy snow weather, causing serious damage to the power grid of Hunan, Jiangxi, Guizhou, Zhejiang and other areas. And ice flashover trip appeared in a wide scale, as well as tower collapse, conductor galloping, and power transmission equipment damage accidents, which affected the normal production and life of the people, and caused huge economic losses ${ }^{[1]}$. Substation as a hub for the power grid in power, the stability of it is very important for power system security to protect the surrounding area. Thus, power transmission and transformation equipment anti-icing and de-icing harm technology is in dire need of research.

At present, the power system of ice disaster prevention and control from the technical aspects can be divided into passive anti-icing and active de-icing (including melting ice) two categories. Passive anti-icing technology is generally carried out by natural conditions, such as wind, earth gravity, sunlight and temperature changes ${ }^{[2]}$, etc. Due to the randomness of natural conditions, this kind of anti-icing technology is influenced by region, season and climate. So it is not universal. Different seasons cannot stop the formation of ice, can only play a certain extent to limit the effect of ice damage. With excellent hydrophobic water and low surface energy anti-icing coatings, such as organosilicon compounds, organic fluorine compounds as substrate, the internal use on low surface energy materials filling, process on the surface of the device, can reduce power transmission and transformation equipment icing to a certain extent ${ }^{[3]}$. But deep research shows that the existing water repellent coatings, which can reach a very high surface contact angle, have excellent waterproof performance, but the anti-icing capacity is not strong, especially in the long-term freezing weather, the effect is very poor.

Transmission line de-icing technology is a way to take effective measures, partially or 
completely remove the accumulated ice on the device, after icing on the wire and insulators. There are two typical techniques in this kind of method, one is the mechanical method, that is to produce mechanical force to remove the accumulated ice by various means, and the other is the large current method using heating melting the ice on the wires. Substation, however, suffer from ice making equipment has bigger difference with transmission lines. Transmission lines is mainly wires and suspension insulator need de-icing. But in the substation, the main goal is to prevent the damage of the pillar insulator, the switch equipment and so on. Therefore, strong vibration de-icing and electromagnetic pulse de-icing technology are also difficult to application, manual with high risk and large labor intensity is difficult to live up to its application, but can only as an emergency under certain conditions de-icing measures. While all kinds of large current ice melting method is to solve the transmission line icing damage, cannot be used to melt ice for power equipment, and in most cases, it is also required to operate in the station to adjust the line on the current or load.

From 2008 to 2010, on the base of analyzing of variable electrical equipment icing hazard characteristics and researching existing substation equipment damage icing techniques at home and abroad, de-icing technology such as hot air de-icing method, hot water de-icing method and heat radiation de-icing were put forward by the State Grid Electric Power Research Institute (EPRI).And EPRI had carried on the research of the feasibility and safety of several thermal de-icing methods. If we do not consider the indirect harm caused by frequent tripping, after transmission lines were covered with ice, then we will focus on protecting substation equipment safe operation. Due to the general substation is located in convenient transportation, in which equipment is relatively concentrated and easily access to energy, then melting ice through heat is likely to be a feasible method.

In order to verify the feasibility of the thermal de-icing method and the effect of de-icing, the paper models the process of hot air de-icing process, and the accurate heat transfer formula is obtained. With level $500 \mathrm{KV}$ transformer bushing, switch and circuit breaker as an example for engineering simulation calculation, its de-icing time, energy consumption and energy use efficiency are studied and analyzed

\section{Engineering Calculation Model of the Electric Hot Air De-icing Method}

\section{Ice removal model of hot air jet}

In order to de-icing the object surface ice, hot air de-icing method with high temperature is used. Jet plate and cylinder flow are two typical forms of enhancing heat transfer in the jet flow, and a large number of empirical correlations of the flow and heat transfer are obtained respectively ${ }^{[4-5]}$. The cross section of the bushing shell, the switch and the brake and so on are round. In order to improving the utilization ratio of the energy in the hot air, we reduce the width of the hot air nozzle, thus the flow pattern of the fluid flow pattern is characterized by the flat jet and the flow around the cylinder. In order to make the simulation results accurately, the heat transfer process of the hot cylinder is simulated by using commercial CFD software to predict the heat transfer performance of this heating mode and study on the influence of the structural parameters of the nozzle.

The flow and heat transfer process of the fluid jet in the cylinder surface is very complicated. Even if the two layer and two equation k-epsilon turbulence model is used, the prediction accuracy is still not high. Therefore, this project uses more advanced turbulence model, which has considered the laminar and turbulent transition of SST k-omega turbulence model, and needing to solve four turbulent flow transmission and differential equations.

In order to simplifying the bushing shell, the switch and other equipment for the cylinder, we cover a certain thickness of the ice in the surface. The hot air at 100 degrees expulsion at the speed 
of $15 \sim 20 \mathrm{~m} / \mathrm{s}$ from the nozzle, and washed out of the surface of the cylinder from the side. Because of the existence of temperature difference, heat transfers between hot air and ice layer, in which the surface heat transfer coefficient using $\mathrm{CFD}^{[6-7]}$. After the ice absorbing the heat of the hot air, a part of the ice is cracked and falls off, and the calculation formula does not need to consider this part. Assuming that the water is removed in time, part of the water rise to the temperature of melting into the water after the melting point, it will not affect the back of the process of de-icing. According to the heat flow between the hot air and ice, the ice melting rate can be predicted, and the ice time, energy consumption and energy efficiency of the hot air can be determined.

\section{The steps of calculating the hot air jet de-icing model}

The hot air jet is divided into ice of the bushing shell, the switch and the brake and other surface spray hot air to remove the ice through the horizontal direction. The basic process of the calculation of the de-icing engineering includes the following steps:

(a) Take the arithmetic mean temperature of hot air and ice, that is $\left(t_{\text {ice }}+t_{\text {hotair }}\right) / 2$, as qualitative temperature of hot air, and calculated air coefficient of heat conductivity $\lambda$, viscosity $V$, the Pulang Prandtl number $P_{r}$ and other physical parameters.

(b) Half the thickness of the ice as feature size of hot air jet heat, transfer the hot air flow velocity at the nozzle is the characteristic velocity, the calculation of heat transfer of jet Reynolds number, see Eq. ${ }^{\circ} 1$,

$$
\operatorname{Re}=\frac{u(D+\delta)}{v}
$$

The $D$ and $\delta$ are the cylinder diameter and ice thickness respectively. $u$ is the characteristic velocity, and the hot air flow velocity at the nozzle is taken.

(c) Calculating the convection heat transfer of hot air and ice of the number of $\mathrm{Nu}$. Calculating the $N u$ number of convective heat transfer of hot air and ice layer by CFD value obtained by simulating criterion related formula. The fitting function of $N u$ number and $\mathrm{Re}$ number of $D=300 \mathrm{~mm}, 600 \mathrm{~mm}$ respectively, see in Eq. ${ }^{\circ} 2$ and Eq. 3 respectively (empirical correlation formula has been used for the fitting function, the heat transfer performance of other diameter cylinder is determined by interpolation.

$$
\begin{aligned}
& \left\{\begin{array}{l}
N u=\left(0.02002+0.00603 \exp \left(-\frac{4.49568}{D}\right)\right) R e^{0.025 ;} b=0.25 D \\
N u=\left(0.02097+0.02244 \exp \left(-\frac{4.4625 s}{D}\right)\right) R e^{0.825 ;} ; b=0.5 D \\
N u=\left(0.02144+0.2503 \exp \left(-\frac{5.0045 \mathrm{D}}{D}\right)\right) R e^{0.825} ; b=1.00 D
\end{array}\right.
\end{aligned}
$$



Heat transfer coefficient h of ice surface can be calculated by Eq. ${ }^{\circ} 4$.

$$
h=\frac{N u \cdot \lambda}{D+\delta}
$$

(d) Heat flux of convective heat transfer between hot and ice per unit time is related to the temperature difference between hot and ice-related. According to the calculation of the cooling formula of Newton, that is

$$
q=h F\left(t_{i \in e}-t_{\text {hotair }}\right)
$$


Where $F$ is the heat transfer area of the hot air and ice. If a given hot air vent area is $A$, and the width is $B$, the length of the rectangular nozzle is $A / b$. Heat transfer area is $. F=\frac{A}{b} \pi(D+\delta)$.

(e) Calculation of the total heat absorption of the process of the hot air in the cylinder The total mass of the ice layer on the cylinder surface can be calculated by the formula Eq. ${ }^{\circ} 6$,

$$
m_{i \sigma o}=\rho_{i \sigma e} \pi L \delta(D+\delta)
$$

Among them, $p_{\text {ieg }}$ the density of ice, $L$ for the bushing shell, the length of the ice body, such as the bushing shell, the switch and the brake.

The actual process is that part of the ice will be detached from the ice before completely thawed. Suppose that the proportion of ice volume which is completely melted represented by $\xi$, the total amount of the melt and the non-melted ice is mice $\xi$ and $(1-\xi)$ mice. The former need to absorb the latent heat, the latter does not need.

Suppose melting ice have become $0^{\circ} \mathrm{C}$ water, and the average temperature of the ice which is not melted off is 0.5 tice. The total heat absorbed by the ice in the process of de-icing is calculated by the formula Eq. ${ }^{0} 7$,

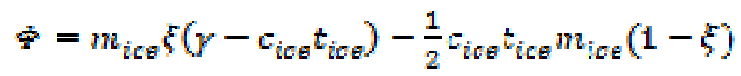

Among them, $c_{i c e}$ is the specific heat of ice, and $\gamma$ is the latent heat of melting ice.

(f) Using the formula Eq. ${ }^{0} 5$ and Eq. ${ }^{\circ}$, we can calculate the total time required by hot air jets de-icing through formula Eq. ${ }^{\circ} 8$

$$
\tau=
$$

(g) It is assumed that the hot air is heated by the wind, and the total energy consumption of the hot air in the air is calculated by the formula Eq. ${ }^{\circ}$.

$$
E_{\text {oon }}=u A \rho_{\text {air }} c_{\text {air }} \tau\left(t_{\text {hotai } r}-t_{\text {sur }}\right)
$$

$t_{\text {sur }}$ is the ambient air temperature, which is equivalent to the initial temperature of the ice layer. In theory, removing the ice surface ice completely and melting the ice by absorbing heat for $E_{\text {nefd }}=m_{i c \varepsilon}\left(\gamma-c_{i c \varepsilon} t_{i c g}\right)$, calculating the energy efficiency of hot air jet in formula Eq. ${ }^{\circ} 10$.

$$
\eta=\frac{\underline{E}_{i: E+L l}}{E_{\mathrm{vQn}}}
$$

\section{Engineering Calculation on the De-icing Performance of Substation Equipment such as Transformer Bushing shell, Switches and Circuit Breakers}

For the characteristics of $500 \mathrm{kv}$ bushing shell, switch and circuit breaker, the engineering simulation is carried out, and the research and analysis are carried out on the de-icing time, energy consumption and energy utilization efficiency. The paper introduces the working conditions and the results of the simulation calculation of the transformer bushing shell.

Calculation on the working condition of bushing shell of transformer.

The bushing shell for 500kv transformer under study includes a horizontally mounted oil bushing CRW-550/1250-3 and cable type transformer oil paper bushing shellBRLW-550/1250-3 which can 
be installed both in horizontal and vertical.

Horizontally mounted oil bushing is shown in Fig. 1. It consists of two parts, indoor (on the right side of the flange) and outdoor (on the left side of the flange). Main geometric dimensions are shown in Table 1. Only take the frozen apart from flange on the left (outdoor) bushing shell surface into consideration in engineering calculation of bushing shell de-icing. Oil paper transformer bushing shell of cable type is shown in Fig. 2, and the main geometric dimensions are shown in Table 3.In the calculation of the bushing shell de-icing, the ice on both side of flange is needed to be considered at the same time.

Table 1 Size of horizontal mounted oilpaper wall bushing

Table 2 the geometry of horizontal / vertical mounted through cable type transformer bushing of oilpaper

\begin{tabular}{|c|c|c|c|c|c|c|c|c|c|c|}
\hline Type & $\mathrm{d}$ & $\mathrm{L}_{1}$ & $\mathrm{~L}_{2}$ & $\mathrm{~L}_{4}$ & $\mathrm{~L}$ & Type & $\mathrm{d}$ & $\mathrm{L}_{1}$ & $\mathrm{~L}_{2}$ & $\mathrm{~L}$ \\
\hline $\begin{array}{c}\text { CRW-55 } \\
0 / 1250-3\end{array}$ & 500 & 4100 & 4000 & 370 & 10020 & $\begin{array}{c}\text { BRLW-5 } \\
50 / 1250-3\end{array}$ & 520 & 4250 & 2415 & 7815 \\
\hline
\end{tabular}

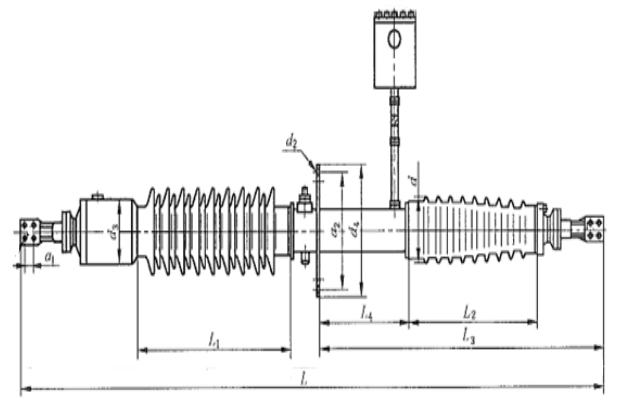

Figure 1 horizontal mounting structure oil wall bushing

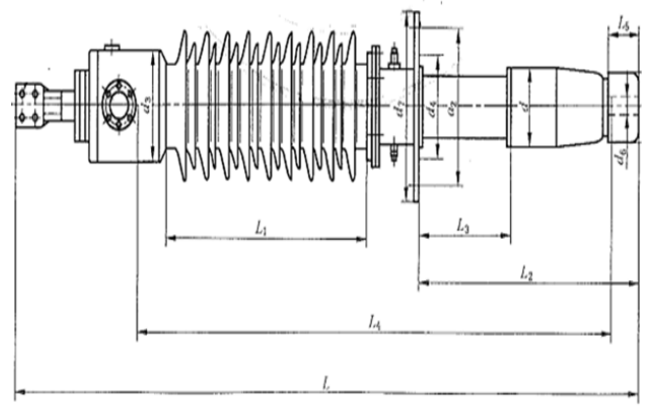

Figure 2 Bushing shell form structure of Greaseproof paper through cable type transformers 
In the work of de-icing calculation, we simplify the bushing shell into cylinder with different length and diameter, which are shown in Table 3. The surface of the cylinder is covered by ice with a thickness of $10 \mathrm{~mm}$.

Table 3 The simplified model of the calculation of the de-icing of transformer bushing shell

\begin{tabular}{ccccl}
\hline Name & Type & $\begin{array}{c}\text { Simplified } \\
\text { length }(\mathrm{mm})\end{array}$ & $\begin{array}{c}\text { Simplified } \\
\text { diameter(mm) }\end{array}$ & Mounted type \\
\hline $\begin{array}{c}\text { 500kv } \\
\text { bushing }\end{array}$ & BRW-550/1250-3 & 4725 & 600 & horizontal mounted \\
\cline { 2 - 5 } & BRL-550/1250-3 & $4575+2893(7468)$ & 600 & $\begin{array}{l}\text { horizontal/vertical } \\
\text { mounted }\end{array}$ \\
\hline
\end{tabular}

Hot air de-icing uses the method of heating the air. When the de-icing is calculated, assume that the ambient temperature is $-8^{\circ} \mathrm{C}$, and the relative humidity is $50 \%$, the velocity is $6 \mathrm{~m} / \mathrm{s}$, and the axis is vertical. Engineering calculation control parameters are shown in Table 4.

Table 4 calculation and control parameters of bushing shell except for ice

\begin{tabular}{|c|c|c|c|c|c|}
\hline $\begin{array}{l}\text { De-icing } \\
\text { method }\end{array}$ & control parameters & 1 & 2 & 3 & Remarks \\
\hline \multirow{6}{*}{$\begin{array}{l}\text { Hot air } \\
\text { de-icing }\end{array}$} & Hot air flow pattern & Jet & & & \\
\hline & Temperature $\left({ }^{\circ} \mathrm{C}\right)$ & 100 & 120 & & \\
\hline & $\operatorname{Velocity}(\mathrm{m} / \mathrm{s})$ & 10 & 15 & 1 & \\
\hline & Nozzle section $\left(\mathrm{cm}^{2}\right)$ & 379.9 & & & $\begin{array}{l}\text { Equivalent to a circle } \\
\text { of diameter } 22 \mathrm{~cm}\end{array}$ \\
\hline & $\begin{array}{l}\text { Dimensionless width of } \\
\text { nozzle }\end{array}$ & 0.25 & 0.5 & & $\begin{array}{c}\text { The cylinder } \\
\text { diameter is the basis }\end{array}$ \\
\hline & $\begin{array}{c}\text { Dimensionless distance of } \\
\text { nozzle and bushing }\end{array}$ & 0.2 & 1.0 & & $\begin{array}{c}\text { The cylinder } \\
\text { diameter is the basis }\end{array}$ \\
\hline
\end{tabular}

Note: there are 2 kinds of distance from the nozzle. In order to make the results more general, the nozzle width and the distance between the nozzles and bushing are non- dimensional.

\section{Calculation results on 500kv transformer bushing shell}

According to the parameters of Table 4, the performance of hot air is simulated, and is shown in Table 5. The energy consumption, de-icing time and energy utilization efficiency of the bushing hot air de-icing are given in Fig. 3.

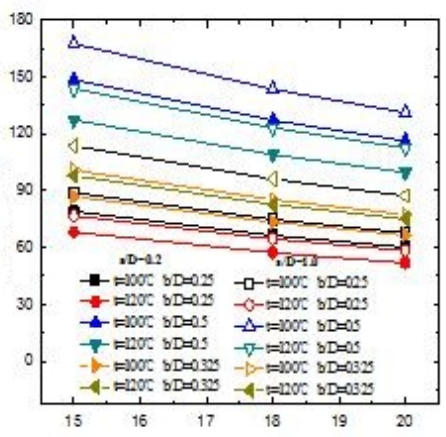

(a)Energy consumption

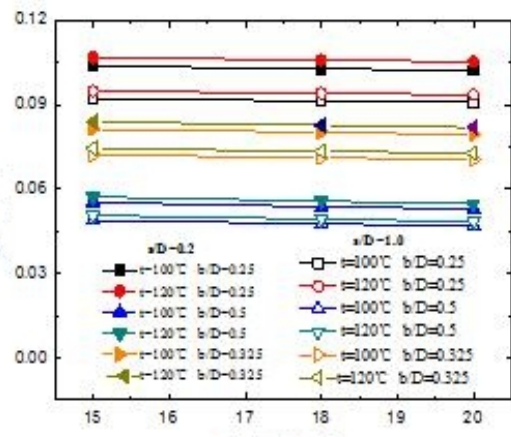

(b) De-icing time

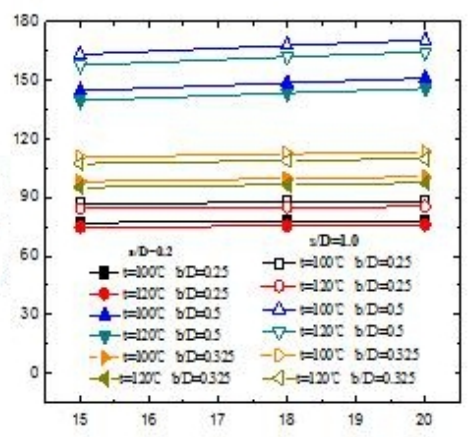

(c) Energy use efficiency

NOTE: in Fig. $3 s / D$ refers to dimensionless distance of nozzle-bushing dimensionless, while $b / D$ refers to dimensionless nozzle width. $D=600 \mathrm{~mm}$.

From Table 5 and Fig. 3 it can be seen that as the wind speed increases, de-icing time reduces, 
as well as energy utilization efficiency, while the energy consumption increases. As outlet temperature increases, de-icing time reduces, as well as the energy consumption, while energy efficiency increases. For three nozzle size with nearly equal cross-sectional area, its de-icing performance is roughly different. Take export wind speed of $18 \mathrm{~m} / \mathrm{s}$, hot air temperature of $100{ }^{\circ} \mathrm{C}$ for example, from Figure 3-3 it is apparent that compared to non-dimensional nozzle width 0.25 , then the dimensionless nozzle width of are 0.325 and 0.5 , the de-icing time grows by $32.7 \%, 91.1 \%$, energy consumption increased by $28.2 \%, 91.1 \%$, and energy use efficiency decreased $22.3 \%, 47.6 \%$. It is easy to draw that when nozzle length is $253 \mathrm{~mm}$ and width is $150 \mathrm{~mm}$, de-icing performance is best. It is because the flow area equals to the rectangular vents, length of nozzle with width 0.25 is double of it of nozzle with width 0.5. Contact area of hot air and the ice doubles too. In accordance with Newton's cooling equation, when two vents hot air temperature and speed are the same, the former transfers double heat than the latter during per unit time. Under the circumstances of same ice thickness (that is the de-icing load), de-icing time is reduced by about half.

Looking at Table 5 and Fig. 3, you can see a small width spout $(b / D=0.25$, namely $253 \mathrm{~m} \times$ $150 \mathrm{~mm}$ spout), small nozzle-bushing distance $(s / D=0.2$, that is, absolute distance $120 \mathrm{~mm})$ under high temperature $\left(120^{\circ} \mathrm{C}\right)$, big speed $(20 \mathrm{~m} / \mathrm{s})$ jet bushing, required less de-icing time $(51.91 \mathrm{~min}$.). If consider energy utilization efficiency the de-icing process, air flow rate $(15 \mathrm{~m} / \mathrm{s})$ can be reduced. This time the corresponding energy consumption and energy utilization efficiency are $74.82 \mathrm{kw} \cdot \mathrm{h}$ and $10.7 \%$, respectively.

Table 5 Calculation results of the de-icing of the $500(\mathrm{kv})$ transformer bushing shell

\begin{tabular}{|c|c|c|c|c|c|c|c|c|c|c|c|c|c|c|}
\hline \multirow{2}{*}{\multicolumn{3}{|c|}{$\begin{array}{c}\text { Dimensionless Nozzle Position } \\
\text { Dimensionless Nozzle Width }\end{array}$}} & \multicolumn{6}{|c|}{0.20 , Corresponding nozzle-Sleeve distance } & \multicolumn{6}{|c|}{1.00, Corresponding nozzle-Sleeve distance } \\
\hline & & & \multicolumn{2}{|c|}{0.25} & \multicolumn{2}{|c|}{0.50} & \multicolumn{2}{|c|}{0.325} & \multicolumn{2}{|c|}{0.25} & \multicolumn{2}{|c|}{0.50} & \multicolumn{2}{|c|}{0.325} \\
\hline \multicolumn{3}{|c|}{ Nozzle Section Size(mm) } & \multicolumn{2}{|c|}{ Length } & \multicolumn{2}{|c|}{ L 126 , W 300} & \multicolumn{2}{|c|}{ L 195, W 195} & \multicolumn{2}{|c|}{ L253, W 150} & \multicolumn{2}{|c|}{ L $126, W 300$} & \multicolumn{2}{|c|}{ L 195, W 195} \\
\hline \multicolumn{3}{|c|}{ Hot Air Temperature $\left({ }^{\circ} \mathrm{C}\right)$} & 100.0 & 12 & & 120.0 & 100.00 & 120.0 & 100.0 & 120.00 & 100.0 & 120.00 & 100.0 & 120.0 \\
\hline Nozzle & 15.0 & De-icing & 79.10 & 68.23 & 148.4 & 127.3 & 100.8 & 86.83 & 89.03 & 76.80 & 167.5 & 143.72 & 113.6 & 97.82 \\
\hline Volocity $(\mathrm{m} / \mathrm{s}$ & 18.0 & De-icing & 66.52 & 57.38 & 127.1 & 109.0 & 85.28 & 73.44 & 74.87 & 64.59 & 143.4 & 123.0 & 96.08 & 82.73 \\
\hline ) & 20.0 & De-icing & 60.18 & 51.91 & 116.2 & 99.70 & 77.41 & 66.66 & 67.74 & 58.43 & 131.2 & 112.54 & 87.21 & 75.10 \\
\hline Nozzle & 15.0 & EngCspt(kw.h) & 77.06 & 74.82 & 144.6 & 139.6 & 98.24 & 95.21 & 86.74 & 84.21 & 163.2 & 157.59 & 110.6 & 107.2 \\
\hline \multirow{2}{*}{ Volocity $(\mathrm{m} / \mathrm{s}$} & 18.0 & EngCspt(kwh) & 77.77 & 75.50 & 148.6 & 143.4 & 99.70 & 96.63 & 87.53 & 84.98 & 167.7 & 161.96 & 112.3 & 108.8 \\
\hline & 20.0 & EngCspt(kwh) & 78.18 & 75.90 & 150.9 & 145.7 & 100.5 & 97.46 & 87.99 & 85.43 & 170.4 & 164.54 & 113.2 & 109.8 \\
\hline Nozzle & 15.0 & EngUtleff & 0.104 & 0.107 & 0.055 & 0.057 & 0.081 & 0.083 & 0.092 & 0.095 & 0.049 & 0.051 & 0.072 & 0.074 \\
\hline \multirow{2}{*}{ Volocity $(\mathrm{m} / \mathrm{s}$} & 18.0 & EngUtleff & 0.103 & 0.106 & 0.054 & 0.056 & 0.080 & 0.082 & 0.091 & 0.094 & 0.048 & 0.049 & 0.071 & 0.073 \\
\hline & 20.0 & EngUtleff & 0.102 & 0.105 & 0.053 & 0.055 & 0.079 & 0.081 & 0.091 & 0.093 & 0.047 & 0.049 & 0.070 & 0.072 \\
\hline
\end{tabular}

Note: EngCspt refers to energy consmption, while EngUtleff refers to energy Utilization efficiency. The data corresponding to the level of 500 kv installation wall bushing CRW-550/1250-3; 500 kv wear BRLW-550/1250-3 cable type transformer oil paper bushing need to consider both sides of the flange of the ice, the energy utilization efficiency and table data are the same, but the de-icing time and energy consurmption is roughly $63 \%$ of the median.

\section{Conclusion}

(1) In this paper, heat characteristics, energy conversion efficiency and energy transmission distance of electric hot air de-icing device are researched. In addition, the accurate formula of convection heat transfer is also derived by establishing the computing model for heat transfer characteristics of hot air de-icing devices used by casing elements, switches and disconnecting link. 
(2) The precise data of de-icing time, energy consumption and energy utilization efficiency were provided through the simulation model established in advance.

(3) According to the result of simulation, electric hot air de-icing device is proved to be feasible and effective in principle. The conclusion is also coincident with the experimental result repeatedly, which means that the devices can be optimized by combining theory with practice.

\section{Reference}

[1]Yi Hu, Analysis and Countermeasures Discussion for Large Area Icing Accident on Power Grid, J. High Voltage Engineering. 2008(2), 34(2), pp.215-219.

[2]Hui Yi, Xingliang Jiang, Transmission line icing and protection, M. Beijing: China Electric Power Press, 2002:192.

[3]Zhicheng Guan, Insulators and power transmission equipment insulation, M. Beijing: Tsinghua University press, 2006: 379.

[4]Xianrong Yang, Qingfang Ma, Radiation heat transfer coefficient manual. Beijing: National Defend Industry Press, 1982:441.

[5]Xinmin Wang, Numerical analysis of ANSYS engineering structure, M. Beijing: China Communications Press, 2007:554.

[6] N. Zuckerman, N. Lior, Radial slot jet impingement flow and heat transfer on a cylindrical target, J. Journal of thermophysics and heat transfer,2007, Vol.21,pp. 548-561.

[7]U. Heck, U. Fritsching, K. Bauckhage, Fluid flow and heat transfer in gas jet quenching of cylinder, J. International Journal of Numerical Methods for Heat \& Fluid Flow,2001, Vol.11(1),pp.36-49. 